\title{
Calorimetric Signature of Deuterated Ice II: Turning an Endotherm to an Exotherm
}

\author{
Violeta Fuentes-Landete, Soroush Rasti, Robert Schlögl, Jörg Meyer, and Thomas Loerting* \\ Cite This: J. Phys. Chem. Lett. 2020, 11, 8268-8274 \\ Read Online
}

ABSTRACT: Calorimetric studies on ice II reveal a surprising $\mathrm{H}_{2} \mathrm{O} / \mathrm{D}_{2} \mathrm{O}$ isotope effect. While the ice II to ice Ic transition is endothermic for $\mathrm{H}_{2} \mathrm{O}$, it is exothermic for $\mathrm{D}_{2} \mathrm{O}$ samples. The transition enthalpies are +40 and $-140 \mathrm{~J} / \mathrm{mol}$, respectively, where such a sign change upon isotope substitution is unprecedented in ice research. To understand the observations we employ force field calculations using two water models known to perform well for $\mathrm{H}_{2} \mathrm{O}$ ice phases and their vibrational properties. These simulations reveal that the isotope effect can be traced back to zero-point energy. q-TIP4P/F fares better and is able to account for approximately three-fourths of the isotope effect, while MB-pol only catches approximately one-third. Phonon and configurational entropy contributions are necessary to predict reasonable transition enthalpies, but they do not have an impact on the isotope effect. We suggest to use these calorimetric isotope data as a benchmark for water models.

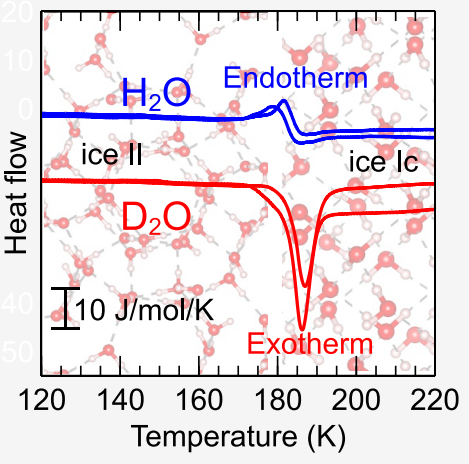

$\mathrm{T}$ he first high-pressure form of ice, ice II, was discovered in 1900 by Tammann. ${ }^{1}$ In the 1960s the structure of ice II was determined to be hydrogen-ordered. ${ }^{2-5}$ Within the framework of the Bernal-Fowler rules ${ }^{6}$ ice II is a phase of zero configurational entropy, where configurational entropy is solely based on the number of microstates differing in terms of the $\mathrm{H}$ atom positions. By contrast, hexagonal ice (ice $\mathrm{Ih}$ ) is geometrically frustrated, showing a disordered network of $\mathrm{H}$ atoms. Ice Ih is hence a phase of nonzero configurational entropy. In fact ice II is thermodynamically more stable than hexagonal ice even at ambient pressure because of its lower entropy, provided the temperature is low. The idea that the high-pressure phase ice II is the ground state at $0 \mathrm{~K}$ and ambient pressure had to be abandoned years later, after the discovery of the hydrogen-ordered counterpart of hexagonal ice, ice XI. ${ }^{7}$ In 1963 Bertie, Calvert, and Whalley examined the transformations that occur when heating high-pressure ices from liquid nitrogen temperatures at ambient pressure. ${ }^{8}$ The thermal analysis was made by using a small silvered-glass vacuum flask as a calorimeter. The heating curve of ice II shows a small break in the heat capacity around 170 to $175 \mathrm{~K}$. Two possible interpretations consistent with the observations were given: (i) A first-order phase transition involving an undetectably small heat, but associated with a large decrease of heat capacity, and (ii) a continuous transition involving a decrease in heat capacity. Twenty-five years later, in 1988, Handa and $\mathrm{Klug}^{9}$ demonstrated that actually interpretation (i) is correct, by detecting the heat involved in the transition to cubic ice (ice Ic). They reported a weak endotherm when heating ice II in their Tian-Calvet calorimeter at $10 \mathrm{~K} / \mathrm{h}$ with an onset temperature at $161 \mathrm{~K}$ and enthalpy of transformation $(\Delta H)$ of $+54 \pm 5 \mathrm{~J} / \mathrm{mol}^{9}{ }^{9}$ That makes ice II the only example of a high-pressure ice converting endothermically to ice Ic at 1 bar. For comparison, other high-pressure ice phases such as ice $\mathrm{V}$, ice VI, or ice XII show pronounced exotherms of $-926 \pm$ $20,{ }^{10}-1523 \pm 16,{ }^{11}$ and $-1233 \pm 23 \mathrm{~J} / \mathrm{mol},{ }^{12}$ respectively.

This is explained by taking into account that the heat of transformation is composed of two contributions: The first contribution is an enthalpic term arising from the transformation from high density (ice II) to low density (ice Ic). The restructuring from ice II to ice Ic involves a density change from 1.21 to $0.94 \mathrm{~g} / \mathrm{cm}^{3} .{ }^{13}$ In spite of this $22 \%$ density decrease, the structural motif of hexagonal channels is conserved. Up to seven-twelfths of all hydrogen bonds are retained at the irreversible cooperative II $\rightarrow$ Ic transition. ${ }^{14}$ Just like for all other high-pressure ice phases this enthalpic term is exothermic. ${ }^{9}$ The second contribution is an entropic term arising from the transformation of hydrogen-ordered (ice II) to hydrogen-disordered (ice Ic). This change in the $\mathrm{H}$ atom subnetwork is also responsible for the decrease in heat capacity observed in the calorimetry scans-the number of degrees of freedom and hence heat capacity is smaller in an H-ordered ice. This entropic term is endothermic, just like for all disordering transformations, for example, melting. In sum the two contributions result in a very weak overall endotherm associated with the ice II $\rightarrow$ ice Ic transformation at 1 bar. By

Received: August 3, 2020

Accepted: September 9, 2020

Published: September 9, 2020 
contrast, ices V, VI, and XII are all hydrogen-disordered. On their phase transitions to hydrogen-disordered ice Ic, only the enthalpic term plays a role, but not the entropic term. The presence of both terms makes the ice II $\rightarrow$ ice Ic transition a particularly challenging case and also of great interest for simulations and benchmarking water models.

Studies with deuterated samples are of high importance, since these are required to deduce the crystal structure from neutron diffraction data. The thermal behavior of several deuterated ice samples has been investigated by differential scanning calorimetry (DSC). ${ }^{11,12,15-17}$ Deuteration usually translates into a more exothermic transformation to ice Ic. Specifically, the exotherm increases by 107 and $175 \mathrm{~J} / \mathrm{mol}$ after deuteration of ices VI and XII, respectively. ${ }^{11,12}$ Although ice II was discovered more than a century ago, no calorimetric data for $\mathrm{D}_{2} \mathrm{O}$ ice II have been available up to now. We here report the thermal behavior of recovered $\mathrm{D}_{2} \mathrm{O}$ ice II and compare it with the one for $\mathrm{H}_{2} \mathrm{O}$. Our study reveals an exothermic ice II to ice Ic transition for $\mathrm{D}_{2} \mathrm{O}$, contrary to the endothermic nature for $\mathrm{H}_{2} \mathrm{O}$. This represents the first example in which the calorimetric signature changes from endothermic to exothermic upon deuteration. To rationalize the finding we analyze the transition based on lattice energy and phonon calculations.

Figure 1 shows representative DSC traces of ice II for $\mathrm{H}_{2} \mathrm{O}$ and $\mathrm{D}_{2} \mathrm{O}$. For each isotopologue two different scans are

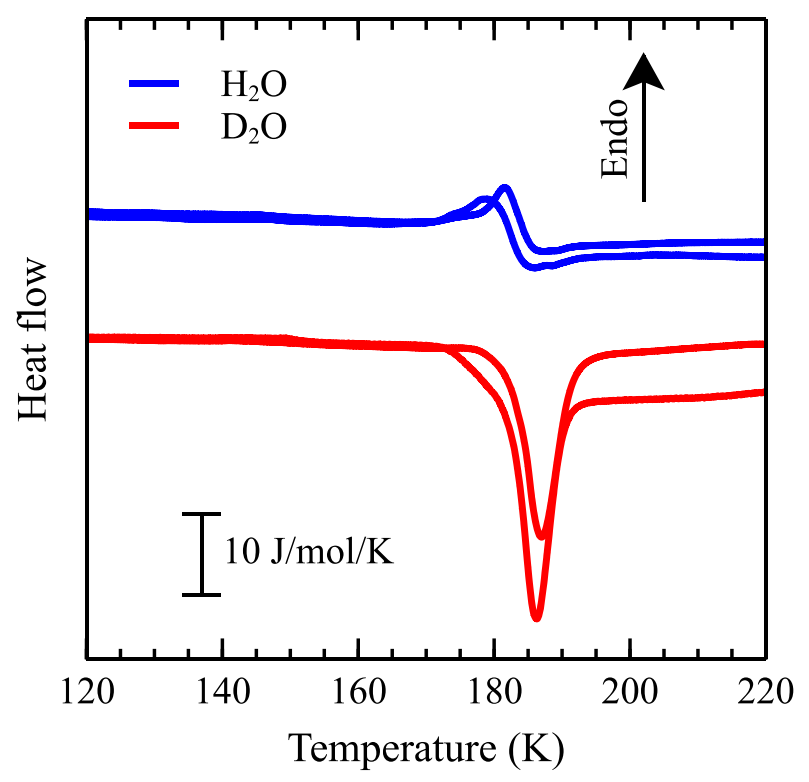

Figure 1. DSC heating scans recorded at $30 \mathrm{~K} / \mathrm{min}$ and ambient pressure for $\mathrm{H}_{2} \mathrm{O}$ (blue) and $\mathrm{D}_{2} \mathrm{O}$ (red) ice II. The signal is normalized to $1 \mathrm{~mol}$. reported, which correspond to the two distinct routes followed for the preparation of the ice II samples (for details, see Experimental and Computational Methods below). The $\mathrm{H}_{2} \mathrm{O}$ calorimetric signature represented here in blue agrees well with the one published by Handa and Klug. ${ }^{9}$ Table 1 shows the enthalpy and onset values calculated in this study for the ice II to ice Ic transition. Note that the heating rate employed by Handa and $\mathrm{Klug}^{9}$ is $10 \mathrm{~K} / \mathrm{h}$, while we employ $30 \mathrm{~K} / \mathrm{min}$, thus explaining the onset temperature shift of $\sim 10 \mathrm{~K}$. The $\mathrm{D}_{2} \mathrm{O}$ scans in Figure 1 in red represent the first measurements for deuterated ice II shown in literature. The exothermic nature of this transition can be easily recognized. Whereas the transition is endothermic for the two preparation protocols for $\mathrm{H}_{2} \mathrm{O}(+33$ \pm 11 and $+48 \pm 9 \mathrm{~J} / \mathrm{mol}$, respectively), it is exothermic for $\mathrm{D}_{2} \mathrm{O}$ ice II $(-132 \pm 36$ and $-149 \pm 50 \mathrm{~J} / \mathrm{mol}$, respectively) (refs 18 and 19). That is, the ice II $\rightarrow$ ice Ic transition is more exothermic by $\sim 180 \pm 20 \mathrm{~J} / \mathrm{mol}$ in $\mathrm{D}_{2} \mathrm{O}$ than in $\mathrm{H}_{2} \mathrm{O}$. In the present work, the enthalpy values are averages of at least 10 different scans for each preparation route. In Figure 1, only one representative DSC scan is shown. This isotope effect on the enthalpy difference to ice Ic is not unusual. Similar differences were found for other high-pressure polymorphs, too. As mentioned above, the transition from ice XII to Ic is $\sim 175 \mathrm{~J} /$ mol more exothermic for $\mathrm{D}_{2} \mathrm{O}$ than for $\mathrm{H}_{2} \mathrm{O}{ }^{12}$

In addition, the onset temperatures for the transition in hydrogenated and deuterated samples are shown in Table 1. The difference in onsets is $7-10 \mathrm{~K}$. This is substantially more than the usual difference between structural transitions in $\mathrm{H}_{2} \mathrm{O}$ and $\mathrm{D}_{2} \mathrm{O}$. For instance, all the triple points in the phase diagram are higher in $\mathrm{D}_{2} \mathrm{O}$ than in $\mathrm{H}_{2} \mathrm{O}$ by between $2.2 \mathrm{~K}$ (for the liquid-ice V-ice VI triple point) and $3.8 \mathrm{~K}$ (for the liquidice Ih-gas triple point). ${ }^{20,21}$ By contrast, dynamic transitions have larger isotope effects. $\mathrm{D}_{2} \mathrm{O}-\mathrm{H}_{2} \mathrm{O}$ isotope effects of $6-8 \mathrm{~K}$ were found for the orientational glass transitions in the $\mathrm{H}$ subnetwork of ices IV, V, VI, and XII. ${ }^{22}$ This suggests that dynamics plays a crucial role for the ice II $\rightarrow$ ice Ic transition as well. While it is $\mathrm{H}$ atom dynamics in the case of the orientational glass transitions, we here measure the isotope effect on the $\mathrm{O}$ atom restructuring. This restructuring takes place in an out-of-equilibrium situation, since ice II is kinetically stable at $77 \mathrm{~K}$ and 1 bar, but not thermodynamically. Similarly, also for the case of the ice XII $\rightarrow$ ice Ic and ice IV $\rightarrow$ ice Ic transitions the isotope effect on the onset temperature amounts to $7 \pm 1 \mathrm{~K}$ at heating rates of $30 \mathrm{~K} /$ min. ${ }^{12}$ Reducing the heating rate to $5 \mathrm{~K} / \mathrm{min}$ the isotope effect is reduced to $4 \pm 1 \mathrm{~K}^{12}$ That is, the onset temperature of the transition is kinetically controlled.

To better understand the observed isotope effect on the transition enthalpy, we performed calculations with two different water models, namely, $\mathrm{q}-\mathrm{TIP} 4 \mathrm{P} / \mathrm{F}^{23}$ and $\mathrm{MB}$ pol. $^{24-26}$ Both of them involve flexible water molecules,

Table 1. Summary ${ }^{a}$

\begin{tabular}{|c|c|c|c|c|c|}
\hline \multirow[b]{2}{*}{ preparation method } & \multirow[b]{2}{*}{ ice II } & \multirow[b]{2}{*}{$T_{0}(\mathrm{~K})$} & \multirow[b]{2}{*}{$\Delta H(\mathrm{~J} / \mathrm{mol})$} & \multicolumn{2}{|c|}{ isotope effect on } \\
\hline & & & & $T_{0}(\mathrm{~K})$ & $\Delta H(\mathrm{~J} / \mathrm{mol})$ \\
\hline Bauer et al. ${ }^{18}$ & $\begin{array}{l}\mathrm{H}_{2} \mathrm{O} \\
\mathrm{D}_{2} \mathrm{O}\end{array}$ & $\begin{array}{l}172 \pm 1 \\
179 \pm 1\end{array}$ & $\begin{array}{l}+33 \pm 11 \\
-132 \pm 36\end{array}$ & $7.0 \pm 1.4$ & $-165 \pm 38$ \\
\hline Salzmann et al. ${ }^{19}$ & $\begin{array}{l}\mathrm{H}_{2} \mathrm{O} \\
\mathrm{D}_{2} \mathrm{O}\end{array}$ & $\begin{array}{l}170 \pm 2 \\
180 \pm 1\end{array}$ & $\begin{array}{l}+48 \pm 9 \\
-149 \pm 50\end{array}$ & $10.0 \pm 2.2$ & $-197 \pm 51$ \\
\hline
\end{tabular}

${ }^{a}$ Summary of the onset temperatures $T_{0}$ and enthalpies $\Delta H$ for $\mathrm{H}_{2} \mathrm{O}$ and $\mathrm{D}_{2} \mathrm{O}$ samples obtained from the DSC measurements for the ice II $\rightarrow$ ice Ic transition following the different preparation routes. 
Table 2. Lattice Energies for Ice Ih and Ice II As Defined in Eqs 1 and (2) ${ }^{a}$

\begin{tabular}{llll} 
& $E_{\text {lat }}$ & & $E_{\text {lat }}^{\text {ZPE }}$ \\
\cline { 2 - 3 } ice Ih & & $\mathrm{H}_{2} \mathrm{O}$ & $\mathrm{D}_{2} \mathrm{O}$ \\
q-TIP4P/F & -59.782 & -47.216 & -49.885 \\
MB-pol & -59.464 & -46.756 & -49.552 \\
experiments & $-58.82^{b}$ & $-47.341 \pm 0.015^{b, c}$ & $-48.611 \pm 0.615^{c}$ \\
ice II & -58.316 & & -49.153 \\
q-TIP4P/F & -58.663 & -46.621 & -48.940 \\
MB-pol & $-58.88 \pm 0.1^{b}$ & $-47.400 \pm 0.1^{b}$ &
\end{tabular}

${ }^{a_{\text {The }}}$ experimental values were obtained by extrapolating experimental values for the vibrational frequencies back to $0 \mathrm{~K}$ and calculating the zeropoint energy therefrom. ${ }^{5,57} .{ }^{b} \operatorname{Ref} 57 .{ }^{c} \operatorname{Ref} 56$.

which allow to calculate vibrational properties including all inter- and intramolecular contributions. We found in previous work that including zero-point energy (ZPE) with q-TIP4P/F provides a very good description of experimental data for a plethora of different crystalline ice phases-outperforming several density functionals despite its simplicity. ${ }^{27}$ Here, we also included the state-of-the-art polarizable many-body force field MB-pol given its excellent description of vibrational properties of ice. ${ }^{28,29}$

We use periodic boundary conditions in order to model the different ice phases and relax the structures while constraining the space group symmetry as suggested by Pfrommer et al. ${ }^{30}$ Absolute lattice energies $E_{\text {lat }}$ are calculated as differences of total energies between the ice phase with $N_{\text {ice }}$ molecules in the unit cell after relaxation $\left(E_{\text {ice }}\right)$ and the corresponding constituent molecules at infinite separation $\left(E_{\mathrm{mol}}\right)$.

$$
E_{\text {lat }}=\frac{1}{N_{\text {ice }}} E_{\text {ice }}-E_{\text {mol }}
$$

Calculating the vibrational frequencies then allows to include the missing ZPE effects according to

$$
E_{\text {lat }}^{\mathrm{ZPE}}=\frac{1}{N_{\text {ice }}}\left(E_{\text {ice }}+E_{\text {ice }}^{\mathrm{ZPE}}\right)-\left(E_{\mathrm{mol}}+E_{\mathrm{mol}}^{\mathrm{ZPE}}\right)
$$

To compare with the measured enthalpies for the ice II $\rightarrow$ Ic transition and analyze the isotope effect, we considered the following (free) energy differences, decreasing the number of approximations in each step.

1. Our starting point is the lattice energy difference

$$
\Delta E_{\text {lat }}=E_{\text {lat }}(\mathrm{I})-E_{\text {lat }}(\text { II })
$$

which is of course identical for $\mathrm{H}_{2} \mathrm{O}$ and $\mathrm{D}_{2} \mathrm{O}$.

2. Isotope effects at $T=0 \mathrm{~K}$ are accounted for when including the (slightly) different ZPE corrections to the lattice energies for each ice phase

$$
\Delta E_{\mathrm{lat}}^{\mathrm{ZPE}}=E_{\mathrm{lat}}^{\mathrm{ZPE}}(\mathrm{I})-E_{\mathrm{lat}}^{\mathrm{ZPE}}(\mathrm{II})
$$

3. Since, on the one hand, the ice II $\rightarrow$ ice Ic transformation occurs at ambient pressure in the experiments, the contribution of the $p V$ term in the Gibbs free energy related to the density decrease during the transformation is negligible. On the other hand, $T \gg$ $0 \mathrm{~K}$, and so the temperature dependence of the vibrational contribution to the Gibbs free energy cannot a priori be neglected.

$$
F_{\mathrm{vib}}(T)=\frac{\frac{1}{2} \sum_{i} \hbar \omega_{i}}{E_{\mathrm{ice}}^{\mathrm{ZPE}}}+\frac{1}{\beta} \sum_{i} \ln \left[1-e^{-\beta \hbar \omega_{i}}\right]
$$

where $\beta=\frac{1}{k_{\mathrm{B}} T}$ with the Boltzmann constant $k_{\mathrm{B}}$, and $\omega_{i}$ are the calculated phonon frequencies. This results in

$$
\Delta \tilde{H}(T)=\Delta E_{\text {lat }}+F_{\text {vib }}(T ; \text { I })-F_{\text {vib }}(T ; \text { II })
$$

4. Finally, we also accounted for the configurational entropy $S_{\text {conf }}$ resulting from the number of possible microstates in the $\mathrm{H}$-subnetwork of the ice. This also includes the temperature dependence of the enthalpies according to

$$
\Delta H(T)=\Delta \tilde{H}(T)+T S_{\text {conf }}
$$

We use the expression $R \ln \left(\frac{3}{2}\right)=3.37 \mathrm{~J} \mathrm{~K} / \mathrm{mol}$ for $S_{\text {conf }}$ as given by Pauling ${ }^{31}$ throughout in this work. This expression is based on the number of microstates allowed according to the Bernal-Fowler ice rules, ${ }^{6}$ where only one central water molecule and four tetrahedrally connected water molecules are considered. Considering the actual structure of ice Ih and using a more rigorous approach, the Pauling expression has been corrected to $R \ln (1.507)=3.40 \mathrm{~J} \mathrm{~K} / \mathrm{mol}^{32-35}$ In spite of neglecting long-range effects such as ring closures, the Pauling approach is accurate to better than $1 \%$ for all ice structures. This accuracy is much better than the chemical accuracy aimed for here.

We note that $\Delta H(T=0 \mathrm{~K})=\Delta \tilde{H}(T=0 \mathrm{~K})=\Delta E_{\text {lat }}^{\mathrm{ZPE}}$; that is, eqs $4,(6)$, and (7) are identical at $T=0 \mathrm{~K}$. Furthermore, we evaluated eqs $2,(4),(6)$, and (7) based on the quasi-harmonic approximation (QHA). For $T \gg 0 \mathrm{~K}$ we thus account for the thermal expansion due to the dependence of $\omega_{i}$ on the unit cell volume. The QHA has been very successful to account for nuclear quantum effects in the different ice phases ${ }^{36,37}$ and found to be very accurate for $T \leq 200 \mathrm{~K}$ for ice Ih and ice II. ${ }^{38}$

Lattice energies without and with ZPE for ice Ih and ice II are listed in Table 2. $E_{\text {lat }}$ is identical for $\mathrm{H}_{2} \mathrm{O}$ and $\mathrm{D}_{2} \mathrm{O}$, whereas $E_{\text {lat }}^{\mathrm{ZPE}}$ differs for both isotopologues. Within the experimental uncertainties both models reproduce the available data for $E_{\text {lat }}$ and $E_{\text {lat }}^{\mathrm{ZPE}}$ within $1.5 \mathrm{~kJ} / \mathrm{mol}$, which is much better than chemical accuracy $(4.2 \mathrm{~kJ} / \mathrm{mol})$.

Table 3 compiles the transition enthalpies for the ice II $\rightarrow$ ice Ih transition. On the basis of $E_{\text {lat }}$ alone (given in the first column) the transition is calculated to be strongly exothermic, 
Table 3. Energy Differences for the Ice II $\rightarrow$ Ice I Transition As Defined by Eqs 3, (4), (6), and (7)

\begin{tabular}{|c|c|c|c|c|c|c|c|}
\hline & \multirow[b]{2}{*}{$\Delta E_{\text {lat }}$} & \multicolumn{2}{|c|}{$\Delta E_{\text {lat }}^{\mathrm{ZPE}}$} & \multicolumn{2}{|c|}{$\begin{array}{c}\Delta \tilde{H}(T=150 \\
\mathrm{K})\end{array}$} & \multicolumn{2}{|c|}{$\begin{array}{c}\Delta H(T=150 \\
\mathrm{K})\end{array}$} \\
\hline & & $\mathrm{H}_{2} \mathrm{O}$ & $\mathrm{D}_{2} \mathrm{O}$ & $\mathrm{H}_{2} \mathrm{O}$ & $\mathrm{D}_{2} \mathrm{O}$ & $\mathrm{H}_{2} \mathrm{O}$ & $\mathrm{D}_{2} \mathrm{O}$ \\
\hline q-TIP4P/F & -1466 & -595 & -732 & -627 & -762 & -121 & -256 \\
\hline isotope effect & & & & & & & \\
\hline MB-pol & -801 & -561 & -612 & -563 & -611 & -57 & -105 \\
\hline isotope effect & & & & & & & \\
\hline
\end{tabular}

where $\mathrm{q}-\mathrm{TIP} 4 \mathrm{P} / \mathrm{F}$ predicts a much stronger exothermicity than MB-pol $(-1466$ vs $-801 \mathrm{~J} / \mathrm{mol})$. This alone does not match the experiments, which are weakly endothermic in the $\mathrm{H}_{2} \mathrm{O}$ case and weakly exothermic in the $\mathrm{D}_{2} \mathrm{O}$ case (see Table 1 ). The exothermicity is significantly reduced when including the $\mathrm{ZPE}$ in $\Delta E_{\text {lat }}^{\mathrm{ZPE}}$ (second column in Table 3). Yet, even after consideration of the ZPE the transitions are still clearly exothermic for both models and both isotopes (between -561 and $-732 \mathrm{~J} / \mathrm{mol}$ ). $\mathrm{q}-\mathrm{TIP} 4 \mathrm{P} / \mathrm{F}$ predicts a somewhat stronger influence of ZPE than MB-pol does. Also when including the vibrational correction in $\Delta H$ this picture does not change very much (third column in Table 3). In fact, the vibrational correction at $150 \mathrm{~K}$ is almost identical for both $\mathrm{H}_{2} \mathrm{O}$ and $\mathrm{D}_{2} \mathrm{O}$. It amounts to $\sim 2 \mathrm{~J} / \mathrm{mol}$ for the MB-pol model and $\sim 30 \mathrm{~J} / \mathrm{mol}$ for q-TIP4P/F. The closest match with experiment is reached when also including the Pauling correction for the configurational entropy in $\Delta H$ (last column in Table 3). After this correction the ice II $\rightarrow$ Ic transition is exothermic by $-105 \mathrm{~J} /$ mol for the $\mathrm{D}_{2} \mathrm{O}$ case and the MB-pol model. This is very close to the experimental value given in Table 1 . However, the switch from endothermic to exothermic cannot be accounted for. Both models show the ice II $\rightarrow$ Ic transition to be slightly exothermic for the $\mathrm{H}_{2} \mathrm{O}$ case, by $-57 \mathrm{~J} / \mathrm{mol}$ for the MB-pol model. This transition enthalpy is very close to zero, just like the experimental value, but on the exothermic side. On the one hand, considering the accuracy with which both water models reproduce the lattice energies in Table 2, it is not surprising that they fail to predict the absolute transition enthalpies correctly. On the other hand, the difference that quantifies the isotope effect on the ice II $\rightarrow$ Ic transition introduces some cancellation of errors, which leads to a better agreement with the experimental value of $-180 \pm 20 \mathrm{~J} / \mathrm{mol}$. The $\mathrm{q}-\mathrm{TIP} 4 \mathrm{P} / \mathrm{F}$ model fares somewhat better, predicting an isotope effect of $-135 \mathrm{~J} / \mathrm{mol}$ as opposed to $-48 \mathrm{~J} / \mathrm{mol}$ in case of MB-pol. Since the configurational entropy is exactly the same and the vibrational correction is almost identical for $\mathrm{H}_{2} \mathrm{O}$ and $\mathrm{D}_{2} \mathrm{O}$, they cancel each other when considering the differences of $\Delta \tilde{H}$ and $\Delta H$, which thus yield essentially the same result as $\Delta E_{\text {lat }}^{\mathrm{ZPE}}$. That is, the main reason for the isotope effect noticed in this work is ZPE.

In summary, we report calorimetry scans for the highpressure phase ice II at ambient pressure. These scans feature (i) the transition to cubic ice (ice Ic) at $170 \pm 2 \mathrm{~K}$, (ii) the very subtle transition from ice Ic to hexagonal (ice Ih) near $210 \mathrm{~K}$, and (iii) the melting of ice Ih at $273 \mathrm{~K}$ (not shown in Figure 1). Upon deuteration the onset temperature of the ice II $\rightarrow$ ice Ic transition shifts to $180 \pm 1 \mathrm{~K}$. This shift of $\sim 10 \mathrm{~K}$ is clearly a kinetic effect and reflects the fact that the transition is from kinetically stable ice II to metastable ice Ic at ambient pressure. The enthalpy associated with the transition changes sign upon isotope substitution, from an endotherm of $+40 \mathrm{~J} /$ $\mathrm{mol}$ in the case of $\mathrm{H}_{2} \mathrm{O}$ to an exotherm of $-140 \mathrm{~J} / \mathrm{mol}$ in the case of $\mathrm{D}_{2} \mathrm{O}$. This represents, to the best of our knowledge, the first observation of sign change upon isotope substitution, at least in the thermal study of ice phases. That is, our findings show how isotope effects may change stability and how a metastable material may become stable upon isotope exchange.

Lattice energy calculations based on two flexible water models (q-TIP4P/F and MB-pol) are considered to explain this finding. The observed isotope effect on the enthalpy can be traced back to the difference of lattice energies including ZPE. ZPE alone results in a difference of $137 \mathrm{~J} / \mathrm{mol}$ for $\mathrm{q}-$ $\mathrm{TIP} 4 \mathrm{P} / \mathrm{F}$, which is close to the experimental value of $180 \mathrm{~J} /$ mol. By contrast, MB-pol shows a difference of only $50 \mathrm{~J} / \mathrm{mol}$, clearly underestimating the ZPE. We have also considered the vibrational contribution at $150 \mathrm{~K}$ and the configurational entropy contribution related to the $\mathrm{H}$-order in ice II and $\mathrm{H}$ disorder in ice Ic. Both of these are important for obtaining good values on the absolute transition enthalpy for the ice II $\rightarrow$ ice Ic transition in $\mathrm{H}_{2} \mathrm{O}$. However, these effects are similar for $\mathrm{D}_{2} \mathrm{O}$ and $\mathrm{H}_{2} \mathrm{O}$ and, accordingly, not of relevance in explaining the isotope effect. Future water models are challenged to reproduce the experimentally observed sign change of the ice II $\rightarrow$ ice Ic transition enthalpy. It might be necessary to include zero-point energies of different ice phases directly in the construction of these models. We suggest the isotope effects on the melting transition of ice Ih $(6012 \mathrm{vs} 6280 \mathrm{~J} / \mathrm{mol})$ and the isotope effects on the transformation from high-pressure ices II (see Table 1), VI, ${ }^{11}$ and $\mathrm{XII}^{12}$ to ice Ic as benchmark data, for which reliable calorimetric studies are available for both $\mathrm{D}_{2} \mathrm{O}$ and $\mathrm{H}_{2} \mathrm{O}$. The ice II $\rightarrow$ ice Ic transition is particularly challenging, because this polymorphic transition involves both entropy and enthalpy changes. They are both of similar size but opposed sign, and so it is highly challenging to reproduce whether an endotherm or an exotherm results in sum.

\section{EXPERIMENTAL AND COMPUTATIONAL METHODS}

The ice II samples were prepared in our piston-cylinder apparatus by using a computerized universal testing machine (Zwick, model BZ100/TL3S). We used $600 \mu \mathrm{L}$ of $\mathrm{H}_{2} \mathrm{O}$ or $\mathrm{D}_{2} \mathrm{O}$, which was pipetted into an indium container inside the high-pressure apparatus for compression. Indium containers were employed to avoid unwanted pressure drops during experiments. ${ }^{39}$ Two different protocols were followed for the preparation of ice II samples. The first protocol is taken from Bauer et al. ${ }^{18}$ and consists of the direct polymorphic transformation of ice Ih to ice II by slow compression to $0.40 \mathrm{GPa}$ at $198 \mathrm{~K}$. The rather low compression rate of 10 $\mathrm{MPa} / \mathrm{min}$ between 0.20 and $0.40 \mathrm{GPa}$ ensures that no parallel polymorphic transformation takes place. ${ }^{18}$ The second protocol used to produce ice II is adapted from the work of Salzmann et al. ${ }^{19}$ By heating ice Ih at $0.50 \mathrm{GPa}$, a chain of polymorphic transformations to ice III, ice II, and ice V takes place up to $\sim 250 \mathrm{~K}$. By the isobaric heating of $\mathrm{Ih}$ at $0.50 \mathrm{GPa}$ to $\sim 200 \mathrm{~K}$ ice II is obtained.

In both routes the pressurized ice II sample is then quenched in liquid nitrogen, and the pressure is released to recover ice II at ambient pressure. No transformations take place upon quench-recovery. In spite of hexagonal ice being the stable phase at $77 \mathrm{~K}$, no transformation back to hexagonal ice takes place while storing the sample at ambient pressure and $77 \mathrm{~K}$. At $77 \mathrm{~K}$ the kinetics of $\mathrm{O}$-atom restructuring is immeasurably slow. The restructuring of the network of $\mathrm{O}$ atoms only proceeds at measurable rates above $\sim 150 \mathrm{~K}$. 
Typically, a mass of $10-20 \mathrm{mg}$ was cut from the cylindrical ice II sample of $600 \mathrm{mg}$ in total. The grains of ice II were then transferred under liquid nitrogen into an aluminum crucible and covered with a lid. These crucibles were cold-loaded into our differential scanning calorimeter (DSC 8000 PerkinElmer) and subsequently heated two times at $30 \mathrm{~K} / \mathrm{min}$. The first scan, heated to $253 \mathrm{~K}$, shows the polymorphic transitions first to ice Ic and ultimately yielding hexagonal ice Ih. The second one, to $313 \mathrm{~K}$, shows the endotherm due to the melting of hexagonal ice Ih. From the melting peak we calculate the mass of the sample based on the known heat of fusion of $\mathrm{D}_{2} \mathrm{O}, 6280 \mathrm{~J} /$ $\mathrm{mol}^{40}$ (or $6012 \mathrm{~J} / \mathrm{mol}$ in the case of $\mathrm{H}_{2} \mathrm{O}$ ). All calorimetry scans were recorded at ambient pressure. This procedure is identical to the established procedure reported in earlier work from our group. ${ }^{22,41,42}$

The computational setup is almost identical to the one used in ref 27 . Briefly, the $\mathrm{q}$-TIP4P/F water model $^{23}$ has been evaluated using the Atomic Simulation Environment (ASE) ${ }^{43}$ with the Lammps code ${ }^{44}$ via the available calculator, whereas a new calculator has been implemented in order to interface with the MB-pol model as developed and implemented by the Paesani group. ${ }^{24-26}$ Data obtained from neutron diffraction experiments ${ }^{45,46}$ have been used with the Genice package ${ }^{47}$ in order to generate the initial structures of ice II and I. ASE is employed for all geometry optimizations with a tight maximum force threshold of $10^{-4} \mathrm{eV} / \AA \AA$. Vibrational properties have been calculated for the different ice phases based on the Parlinski-LiKawazoe finite-displacement method ${ }^{48}$ (using displacements of $10^{-3} \AA$ ) as implemented in the phonopy package. ${ }^{49}$ Very tight $30 \times 30 \times 30$ reciprocal space grids were used for the evaluation of eq 5 , resulting in at least 1456 points in the irreducible wedge of the Brillouin zone for which vibrational frequencies were calculated.

In our calculations we used ice Ih in place of ice Ic, since these two ice phases are energetically almost degenerate, with hexagonal ice being more stable by a margin close to zero, even difficult to measure in calorimetry scans. Figure 1 shows a very small baseline change near $210 \mathrm{~K}$ that corresponds to the ice Ic to ice Ih transition-this exotherm is smaller than $10 \mathrm{~J} / \mathrm{mol}$ and negligible in the present context. Engel et al. ${ }^{50}$ have suggested that the proper energetic ordering of ices Ih and Ic can only be described by taking anharmonic affects into account, which is beyond the scope of the present work. Furthermore, ice Ic and ice Ih have almost indistinguishable spectral properties from the microwave to the ultraviolet. For this reason they are commonly both denoted as ice I, where the difference between ice Ic and ice Ih is the stacking sequence. Specifically, all vibrational transitions have long been thought to be identical for ice Ic and ice Ih. ${ }^{51}$ Also the intermolecular phonon modes in the far-infrared spectral range barely reveal any difference. ${ }^{52,53}$ Subtle differences based on different polytypes of ice I containing different hexagonal and cubic stacking sequences have only been noted recently by Carr et al. ${ }^{54}$ In a very recent work the transition from a pure cubic stacking sequence to a pure hexagonal stacking sequence was identified, where only very small shifts, for example, of $\sim 2$ $\mathrm{cm}^{-1}$ in the translational band mark the transition. ${ }^{55}$

\section{ASSOCIATED CONTENT}

\section{SI Supporting Information}

The Supporting Information is available free of charge at https://pubs.acs.org/doi/10.1021/acs.jpclett.0c02368.
Illustrated structures (ZIP)

Space-group-constrained structure optimizations, calculated thermal expansion (PDF)

\section{AUTHOR INFORMATION}

\section{Corresponding Author}

Thomas Loerting - Institute of Physical Chemistry, University of Innsbruck, 6020 Innsbruck, Austria; (1) orcid.org/0000-

0001-6694-3843; Email: thomas.loerting@uibk.ac.at

\section{Authors}

Violeta Fuentes-Landete - Institute of Physical Chemistry, University of Innsbruck, 6020 Innsbruck, Austria; Max Planck Institute for Chemical Energy Conversion, 45470 Mülheim an der Ruhr, Germany

Soroush Rasti - Leiden Institute of Chemistry, Gorlaeus Laboratories, Leiden University, 2300 RA Leiden, The Netherlands

Robert Schlögl - Max Planck Institute for Chemical Energy Conversion, 45470 Mülheim an der Ruhr, Germany

Jörg Meyer - Leiden Institute of Chemistry, Gorlaeus Laboratories, Leiden University, 2300 RA Leiden, The Netherlands; 이이.0rid.org/0000-0003-0146-730X

Complete contact information is available at:

https://pubs.acs.org/10.1021/acs.jpclett.0c02368

\section{Notes}

The authors declare no competing financial interest.

\section{ACKNOWLEDGMENTS}

We are grateful to the Austrian Science Fund (FWF) for support under the bilateral grant I1392. V.F.L. is grateful to the Max Planck Institute for Chemical Energy Conversion for the financial support. J.M. gratefully acknowledges financial support from The Netherlands Organization for Scientific Research (NWO) under Vidi Grant No. 723.014.009.

\section{REFERENCES}

(1) Tammann, G. Ueber die Grenzen des festen Zustandes V. Ann. Phys. 1900, 308, 161-194.

(2) Wilson, G. J.; Chan, R. K.; Davidson, D. W.; Whalley, E. Dielectric Properties of Ices II, III, V, and VI. J. Chem. Phys. 1965, 43, 2384-2391.

(3) Bertie, J. E.; Whalley, E. Infrared Spectra of Ices II, III, and V in the Range 4000 to $350 \mathrm{~cm}^{-1}$. J. Chem. Phys. 1964, 40, 1646-1659.

(4) Finch, E. D.; Rabideau, S. W.; Wenzel, R. G.; Nereson, N. G. Neutron-Diffraction Study of Ice Polymorphs. II. Ice II. J. Chem. Phys. 1968, 49, 4361-4365.

(5) Kamb, B.; Hamilton, W. C.; LaPlaca, S. J.; Prakash, A. Ordered Proton Configuration in Ice II, from Single-Crystal Neutron Diffraction. J. Chem. Phys. 1971, 55, 1934-1945.

(6) Bernal, J. D.; Fowler, R. H. A Theory of Water and Ionic Solution, with Particular Reference to Hydrogen and Hydroxyl ions. J. Chem. Phys. 1933, 1, 515-548.

(7) Kawada, S. Dielectric Dispersion and Phase Transition of $\mathrm{KOH}$ Doped Ice. J. Phys. Soc. Jpn. 1972, 32, 1442-1442.

(8) Bertie, J. E.; Calvert, L. D.; Whalley, E. Transformations of Ice II, Ice III, and Ice V at Atmospheric Pressure. J. Chem. Phys. 1963, 38, $840-846$

(9) Handa, Y. P.; Klug, D. D. Heat Capacity and Glass Transition Behavior of Amorphous Ice. J. Phys. Chem. 1988, 92, 3323-3325.

(10) Salzmann, C. G.; Kohl, I.; Loerting, T.; Mayer, E.; Hallbrucker, A. The Low-Temperature Dynamics of Recovered Ice XII as Studied by Differential Scanning Calorimetry: A Comparison with Ice V. Phys. Chem. Chem. Phys. 2003, 5, 3507-3517. 
(11) Shephard, J. J.; Salzmann, C. G. The Complex Kinetics of the Ice VI to Ice XV Hydrogen Ordering Phase Transition. Chem. Phys. Lett. 2015, 637, 63-66.

(12) Salzmann, C. G.; Mayer, E.; Hallbrucker, A. Thermal Properties of Metastable Ices IV and XII: Comparison, Isotope Effects and Relative Stabilities. Phys. Chem. Chem. Phys. 2004, 6, 1269-1276.

(13) Loerting, T.; Bauer, M.; Kohl, I.; Watschinger, K.; Winkel, K.; Mayer, E. Cryoflotation: Densities of Amorphous and Crystalline Ices. J. Phys. Chem. B 2011, 115, 14167-14175.

(14) Zheligovskaya, E. A. Structural Mechanisms of the Ih-II and II $\rightarrow$ Ic Transitions between the Crystalline Phases of Aqueous Ice. Crystallogr. Rep. 2015, 60, 714-720.

(15) Shephard, J. J.; Salzmann, C. G. Molecular Reorientation Dynamics Govern the Glass Transitions of the Amorphous Ices. J. Phys. Chem. Lett. 2016, 7, 2281-2285.

(16) Elsaesser, M. S.; Winkel, K.; Mayer, E.; Loerting, T. Reversibility and Isotope Effect of the Calorimetric Glass $\rightarrow$ Liquid Transition of Low-Density Amorphous Ice. Phys. Chem. Chem. Phys. 2010, 12, 708-712.

(17) Johari, G. P.; Hallbrucker, A.; Mayer, E. The Glass-Liquid Transition of Hyperquenched Water. Nature 1987, 330, 552-553.

(18) Bauer, M.; Elsaesser, M. S.; Winkel, K.; Mayer, E.; Loerting, T. Compression-Rate Dependence of the Phase Transition from Hexagonal Ice to Ice II and/or Ice III. Phys. Rev. B: Condens. Matter Mater. Phys. 2008, 77, 220105.

(19) Salzmann, C. G.; Radaelli, P. G.; Finney, J. L.; Mayer, E. A Calorimetric Study on the Low Temperature Dynamics of Doped Ice $\mathrm{V}$ and Its Reversible Phase Transition to Hydrogen Ordered Ice XIII. Phys. Chem. Chem. Phys. 2008, 10, 6313-6324.

(20) Chaplin, M. Water Phase Diagram-Triple Points. http:// www 1.lsbu.ac.uk/water/water phase diagram.html\#trip. Accessed July 31, 2020.

(21) International Association for the Properties of Water and Steam. Revised Release on the Pressure along the Melting and Sublimation Curves of Ordinary Water Substance; International Association for the Properties of Water and Steam, 2011.

(22) Fuentes-Landete, V.; Plaga, L. J.; Keppler, M.; Böhmer, R.; Loerting, T. Nature of Water's Second Glass Transition Elucidated by Doping and Isotope Substitution Experiments. Phys. Rev. X 2019, 9, 011015.

(23) Habershon, S.; Markland, T. E.; Manolopoulos, D. E. Competing Quantum Effects in the Dynamics of a Flexible Water Model. J. Chem. Phys. 2009, 131, 024501.

(24) Babin, V.; Leforestier, C.; Paesani, F. Development of a "First Principles" Water Potential with Flexible Monomers: Dimer Potential Energy Surface, VRT Spectrum, and Second Virial Coefficient. J. Chem. Theory Comput. 2013, 9, 5395-5403.

(25) Babin, V.; Medders, G. R.; Paesani, F. Development of a "First Principles" Water Potential with Flexible Monomers. II: Trimer Potential Energy Surface, Third Virial Coefficient, and Small Clusters. J. Chem. Theory Comput. 2014, 10, 1599-1607.

(26) Medders, G. R.; Babin, V.; Paesani, F. Development of a "FirstPrinciples" Water Potential with Flexible Monomers. III. Liquid Phase Properties. J. Chem. Theory Comput. 2014, 10, 2906-2910.

(27) Rasti, S.; Meyer, J. Importance of Zero-Point Energy for Crystalline Ice Phases: A Comparison of Force Fields and Density Functional Theory. J. Chem. Phys. 2019, 150, 234504.

(28) Moberg, D. R.; Straight, S. C.; Knight, C.; Paesani, F. Molecular Origin of the Vibrational Structure of Ice Ih. J. Phys. Chem. Lett. 2017, $8,2579-2583$.

(29) Moberg, D. R.; Sharp, P. J.; Paesani, F. Molecular-Level Interpretation of Vibrational Spectra of Ordered Ice Phases. J. Phys. Chem. B 2018, 122, 10572-10581.

(30) Pfrommer, B. G.; Côté, M.; Louie, S. G.; Cohen, M. L. Relaxation of Crystals with the Quasi-Newton Method. J. Comput. Phys. 1997, 131, 233-240.

(31) Pauling, L. The Structure and Entropy of Ice and of Other Crystals with Some Randomness of Atomic Arrangement. J. Am. Chem. Soc. 1935, 57, 2680-2684.
(32) Nagle, J. Lattice Statistics of Hydrogen Bonded Crystals. I. The Residual Entropy of Ice. J. Math. Phys. 1966, 7, 1484-1491.

(33) Berg, B. A.; Muguruma, C.; Okamoto, Y. Residual Entropy of Ordinary Ice from Multicanonical Simulations. Phys. Rev. B: Condens. Matter Mater. Phys. 2007, 75, 092202.

(34) Herrero, C. P.; Ramírez, R. Configurational Entropy of Hydrogen-Disordered Ice Polymorphs. J. Chem. Phys. 2014, 140, 234502.

(35) Ferreyra, M. V.; Grigera, S. A. Boundary Conditions and the Residual Entropy of Ice Systems. Phys. Rev. E: Stat. Phys., Plasmas, Fluids, Relat. Interdiscip. Top. 2018, 98, 042146.

(36) Pamuk, B.; Soler, J. M.; Ramírez, R.; Herrero, C. P.; Stephens, P. W.; Allen, P. B.; Fernández-Serra, M.-V. Anomalous Nuclear Quantum Effects in Ice. Phys. Rev. Lett. 2012, 108, 193003.

(37) Umemoto, K.; Sugimura, E.; de Gironcoli, S.; Nakajima, Y.; Hirose, K.; Ohishi, Y.; Wentzcovitch, R. M. Nature of the Volume Isotope Effect in Ice. Phys. Rev. Lett. 2015, 115, 173005.

(38) Ramírez, R.; Neuerburg, N.; Fernández-Serra, M.-V.; Herrero, C. P. Quasi-Harmonic Approximation of Thermodynamic Properties of Ice Ih, II, and III. J. Chem. Phys. 2012, 137, 044502.

(39) Mishima, O.; Calvert, L.; Whalley, E. 'Melting Ice' I at $77 \mathrm{~K}$ and 10 kbar: A New Method of Making Amorphous Solids. Nature 1984, 310, 393-395.

(40) Long, E.; Kemp, J. The Entropy of Deuterium Oxide and the Third Law of Thermodynamics. Heat Capacity of Deuterium Oxide from 15 to $298 \mathrm{~K}$. The Melting Point and Heat of Fusion. J. Am. Chem. Soc. 1936, 58, 1829-1834.

(41) Gasser, T. M.; Thoeny, A. V.; Plaga, L. J.; Köster, K. W.; Etter, M.; Böhmer, R.; Loerting, T. Experiments Indicating a Second Hydrogen Ordered Phase of Ice VI. Chem. Sci. 2018, 9, 4224-4234.

(42) Fuentes-Landete, V.; Köster, K. W.; Böhmer, R.; Loerting, T. Thermodynamic and Kinetic Isotope Effects on the Order-Disorder Transition of Ice XIV to Ice XII. Phys. Chem. Chem. Phys. 2018, 20, 21607-21616.

(43) Hjorth Larsen, A.; Jorgen Mortensen, J.; Blomqvist, J.; Castelli, I. E.; Christensen, R.; Dułak, M.; Friis, J.; Groves, M. N.; Hammer, B.; Hargus, C.; et al. The Atomic Simulation Environment-a Python Library for Working with Atoms. J. Phys.: Condens. Matter 2017, 29, 273002.

(44) Plimpton, S. Fast Parallel Algorithms for Short-Range Molecular Dynamics. J. Comput. Phys. 1995, 117, 1-19.

(45) Lobban, C.; Finney, J. L.; Kuhs, W. F. The $p-T$ Dependency of the Ice II Crystal Structure and the Effect of Helium Inclusion. J. Chem. Phys. 2002, 117, 3928-3934.

(46) Fortes, A. D. Accurate and Precise Lattice Parameters of $\mathrm{H}_{2} \mathrm{O}$ and $\mathrm{D}_{2} \mathrm{O}$ Ice Ih between 1.6 and $270 \mathrm{~K}$ from High-Resolution Timeof-Flight Neutron Powder Diffraction Data. Acta Crystallogr., Sect. B: Struct. Sci., Cryst. Eng. Mater. 2018, 74, 196-216.

(47) Matsumoto, M.; Yagasaki, T.; Tanaka, H. GenIce: HydrogenDisordered Ice Generator. J. Comput. Chem. 2018, 39, 61-64.

(48) Parlinski, K.; Li, Z. Q.; Kawazoe, Y. First-Principles Determination of the Soft Mode in Cubic $\mathrm{ZrO}_{2}$. Phys. Rev. Lett. 1997, 78, 4063-4066.

(49) Togo, A.; Tanaka, I. First Principles Phonon Calculations in Materials Science. Scr. Mater. 2015, 108, 1-5.

(50) Engel, E. A.; Monserrat, B.; Needs, R. J. Anharmonic Nuclear Motion and the Relative Stability of Hexagonal and Cubic Ice. Phys. Rev. X 2015, 5, 021033.

(51) Taylor, M.; Whalley, E. Raman Spectra of Ices Ih, Ic, II, III, and V. J. Chem. Phys. 1964, 40, 1660-1664.

(52) Li, J. Inelastic Neutron Scattering Studies of Hydrogen Bonding in Ices. J. Chem. Phys. 1996, 105, 6733-6755.

(53) Klug, D. D.; Whalley, E.; Svensson, E. C.; Root, J. H.; Sears, V. F. Densities of Vibrational States and Heat Capacities of Crystalline and Amorphous $\mathrm{H}_{2} \mathrm{O}$ Ice Determined by Neutron Scattering. Phys. Rev. B: Condens. Matter Mater. Phys. 1991, 44, 841-844.

(54) Carr, T. H. G.; Shephard, J. J.; Salzmann, C. G. Spectroscopic Signature of Stacking Disorder in Ice I. J. Phys. Chem. Lett. 2014, 5, $2469-2473$ 
(55) Celli, M.; Ulivi, L.; del Rosso, L. Raman Investigation of the Ice Ic-Ice Ih Transformation. J. Phys. Chem. C 2020, 124, 17135-17140.

(56) Whalley, E. The Difference in the Intermolecular Forces of $\mathrm{H}_{2} \mathrm{O}$ and $\mathrm{D}_{2} \mathrm{O}$. Trans. Faraday Soc. 1957, 53, 1578-1585.

(57) Whalley, E. Energies of the Phases of Ice at Zero Temperature and Pressure. J. Chem. Phys. 1984, 81, 4087-4092. 